\title{
Docente Virtual. Habilidades, Conocimientos y Características.
}

\author{
Virtual Teacher Skills, Knowledge and Characteristics.
}

\author{
Antonio de Jesús Ángeles Villeda ${ }^{a}$
}

\begin{abstract}
:
At present all teachers must possess diverse skills in the use of technological tools and digital applications that allow interaction and effective communication between the teacher and the student, the above, not only focuses on the teacher who works in the virtual environment, but also in the professor who teaches on the face-to-face stage, since he makes continuous use of these support tools in his planning and study program.

Chao (2014) states that the teacher who works in the virtual mode, must have the necessary knowledge in the area taught, as well as master the various techniques and teaching strategies that stimulate learning in the student, also be responsible for planning the formative action in the student, guide, guide and carry out the follow-up in the process of teaching learning and evaluation of tasks.

Teachers of virtual environments must commit themselves to the continuous updating of new tools, because these are modified and changed very quickly. Among the main characteristics and skills that have a virtual teacher are the following: Create and maintain a friendly and social learning community, motivate students so that they do not diminish their commitment, encourage interest and encouragement to stimulate their thinking, interact constantly with the students and carry out a follow-up of all the activities carried out by each of them, design and develop teaching activities or teaching materials that will be used in the course in different multimedia formats, advise the students in the efficient use of the various technological tools for the search and treatment of information and promote autonomous learning - meaningful in the student (Becerril, Sosa, Delgadillo \& Torres, 2015).
\end{abstract}

The following map shows the main characteristics, skills, knowledge and other aspects that a professor must have when he teaches subject using virtual teaching environments:

\section{Keywords:}

Teacher, Virtual, Teaching, Learning, Tools

\section{Resumen:}

En la actualidad todos los profesores deben poseer diversas habilidades en el manejo de herramientas tecnológicas y aplicaciones digitales que permitan la interacción y comunicación efectiva entre el profesor y el alumno, lo anterior, no solo se enfoca en el profesor que labora en el entorno virtual, sino también en el profesor que imparte catedra en el escenario presencial, debido a que este hace uso continuo de estas herramientas de apoyo en su planeación y programa de estudios.

Chao (2014) afirma que el maestro que labora en la modalidad virtual, debe contar los conocimientos necesarios en el área que imparte, así como dominar las diversas técnicas y estrategias didácticas que estimulen el aprendizaje en el alumno, asimismo será el responsable de planear la acción formativa en el estudiante, orientar, guiar y llevar el seguimiento en el proceso de enseñanza aprendizaje y evaluación de tareas.

Los maestros de entornos virtuales deben comprometerse en la actualización continua de nuevas herramientas, debido a que estas se modifican y cambian con gran rapidez. Dentro de las principales características y habilidades a Profesor por Asignatura del Área Académica de Mercadotecnia, Instituto de Ciencias Económico Administrativas, Universidad Autónoma del 
que posee un maestro virtual están las siguientes: Crear y mantener una comunidad de aprendizaje amigable y social, motivar a los alumnos para que no disminuyan su empeño, fomentar el interés y animación para estimular su pensamiento, interactuar constantemente con los alumnos y llevar a cabo un seguimiento de todas las actividades que realice cada uno de ellos, diseñar y desarrollar actividades o materiales didácticos de enseñanza que se utilizaran en el curso en diferentes formatos multimedia, asesorar a los alumnos en el uso eficiente de las diversas herramientas tecnológicas para la búsqueda y tratamiento de la información y promover un aprendizaje autónomo - significativo en el estudiante (Becerril, Sosa, Delgadillo \& Torres, 2015).

El siguiente mapa, muestra las principales características, habilidades, conocimientos y demás aspectos que debe tener un profesor cuando imparte asignatura utilizando entornos de enseñanza aprendizaje virtuales:

\section{Palabras Clave:}

Docente, Virtual, Enseñanza, Aprendizaje, Herramientas 


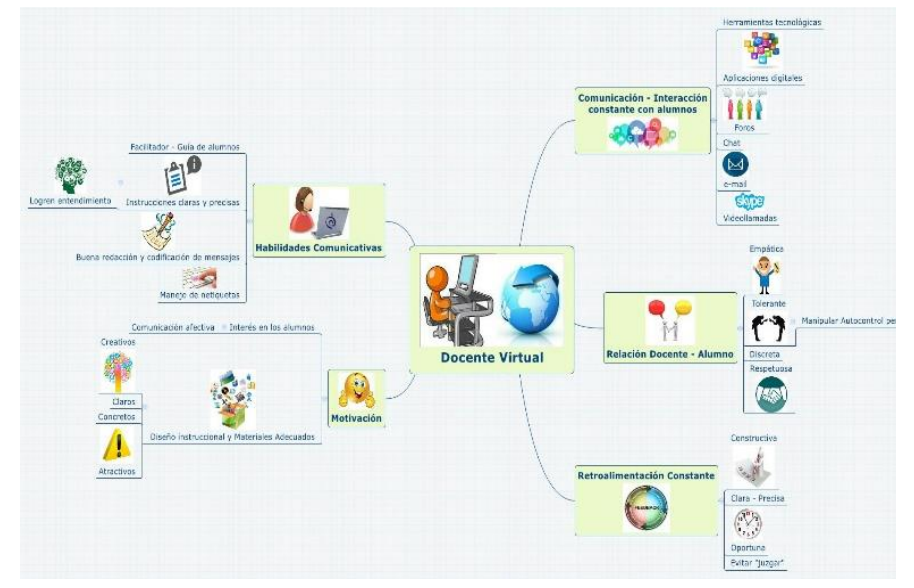

Figura 1. Mapa "Docente Virtual"

\section{Referencias}

[1] Becerril, C., Sosa, G., Delgadillo, M. \& Torres, S. (2015). Competencias Básicas de un Docente Virtual. Revista de Sistemas y Gestión Educativa, 2(4). pp. 882-887. Recuperado de http://www.ecorfan.org/bolivia/researchjour nals/Sistemas y Gestion Educativa/vol2n um4/18.pdf

[2] Chao, M. (2014). El rol del profesor en la educación virtual. Universidad Virtual del Estado de Guanajuato. Revista Iberoamericana para la Investigación y el Desarrollo Educativo. Recuperado de http://www.ride.org.mx/111/index.php/RIDESECUNDARIO/article/vi ewFile/830/811 